\title{
A MASSIVE RUNAWAY STAR FROM 30 DORADUS*
}

\author{
C. J. Evans ${ }^{1}$, N. R. Walborn ${ }^{2}$, P. A. Crowther ${ }^{3}$, V. Hénault-Brunet ${ }^{4}$, D. Massa ${ }^{2}$, W. D. Taylor ${ }^{4}$, I. D. Howarth ${ }^{5}$, \\ H. SANA ${ }^{6,7}$, D. J. LenNON ${ }^{2,8}$, AND J. TH. VAN LOON ${ }^{9}$ \\ ${ }^{1}$ UK Astronomy Technology Centre, Royal Observatory Edinburgh, Blackford Hill, Edinburgh, EH9 3HJ, UK \\ ${ }^{2}$ Space Telescope Science Institute, 3700 San Martin Drive, Baltimore, MD 21218, USA \\ ${ }^{3}$ Department of Physics and Astronomy, University of Sheffield, Hounsfield Road, Sheffield, S3 7RH, UK \\ ${ }^{4}$ Scottish Universities Physics Alliance (SUPA), Institute for Astronomy, University of Edinburgh, Royal Observatory Edinburgh, Blackford Hill, \\ Edinburgh, EH9 3HJ, UK \\ ${ }^{5}$ Department of Physics and Astronomy, University College London, Gower Street, London, WC1E 6BT, UK \\ ${ }^{6}$ European Southern Observatory, Alonso de Cordova 1307, Casilla 19001, Santiago 19, Chile \\ ${ }^{7}$ Sterrenkundig Instituut Anton Pannekoek, Universiteit van Amsterdam, Postbus 94249, 1090 GE Amsterdam, The Netherlands \\ ${ }^{8}$ European Space Agency, Research and Scientific Support Department, 3700 San Martin Drive, Baltimore, MD 21218, USA \\ ${ }^{9}$ Astrophysics Group, School of Physical and Geographical Sciences, Keele University, Staffordshire, ST5 5BG, UK \\ Received 2010 February 24; accepted 2010 April 8; published 2010 May 5
}

\begin{abstract}
We present the first ultraviolet (UV) and multi-epoch optical spectroscopy of 30 Dor 016, a massive O2-type star on the periphery of 30 Doradus in the Large Magellanic Cloud. The UV data were obtained with the Cosmic Origins Spectrograph on the Hubble Space Telescope as part of the Servicing Mission Observatory Verification program after Servicing Mission 4, and reveal \#016 to have one of the fastest stellar winds known. From analysis of the C IV $\lambda \lambda 1548-51$ doublet we find a terminal velocity, $v_{\infty}=3450 \pm 50 \mathrm{~km} \mathrm{~s}^{-1}$. Optical spectroscopy is from the VLT-FLAMES Tarantula Survey, from which we rule out a massive companion (with 2 days $<P<1 \mathrm{yr}$ ) to a confidence of $98 \%$. The radial velocity of $\# 016$ is offset from the systemic value by $-85 \mathrm{~km} \mathrm{~s}^{-1}$, suggesting that the star has traveled the $120 \mathrm{pc}$ from the core of 30 Doradus as a runaway, ejected via dynamical interactions.
\end{abstract}

Key words: open clusters and associations: individual (30 Doradus) - stars: early-type - stars: fundamental parameters - stars: mass-loss

Online-only material: color figures

\section{INTRODUCTION}

30 Doradus in the Large Magellanic Cloud (LMC) is the richest $\mathrm{H}$ II region in the Local Group, providing an excellent template with which to study regions of intense star formation, and both stellar and cluster evolution. It harbors a significant fraction of the most massive and luminous stars known, with a rich population of the earliest O-type stars (e.g., Melnick 1985; Walborn \& Blades 1997), particularly in R136, the dense cluster at its core (Massey \& Hunter 1998).

The O3 spectral class was introduced by Walborn (1971) to accommodate stars in which He I $\lambda 4471$ was absent in moderateresolution photographic spectra, compared to the very weak absorption seen in O4-type spectra. The classification scheme was extended further by Walborn et al. (2002) to include the new types of $\mathrm{O} 2$ and $\mathrm{O} 3.5$ to delineate the behavior of the $\mathrm{N}$ III, $\mathrm{N}$ IV, and $\mathrm{N} \mathrm{v}$ features seen in the earliest types. Even in the age of large multi-object surveys only a few tens of stars are known with $\mathrm{O} 2-\mathrm{O} 3.5$ types. Although very rare, their influence is farreaching as they are expected to evolve rapidly into nitrogen rich Wolf-Rayet stars (WN types), plausible progenitors of supernovae and, potentially, gamma-ray bursts (Smartt 2009).

Observations with the 2-degree Field $(2 \mathrm{dF})$ instrument at the Anglo-Australian Telescope revealed a new O2-type star on the western fringes of 30 Doradus (Figure 1), with a radial velocity of $\sim 85 \mathrm{~km} \mathrm{~s}^{-1}$ lower than the systemic velocity of

\footnotetext{
* Based on observations obtained at the European Southern Observatory Very Large Telescope in program 184.D-0222 and with the NASA/ESA Hubble Space Telescope in program 11484, obtained at the Space Telescope Science Institute, which is operated by the Association of Universities for Research in Astronomy, Inc., under NASA contract NAS 5-26555.
}

nearby massive stars (e.g., Bosch et al. 2009). New multiepoch spectroscopy of this star, 30 Dor 016 in the VLTFLAMES Tarantula Survey (Evans et al. 2010), now enables us to rule out the presence of a close massive companion to a high level of confidence, suggesting that the star might have been ejected from the denser central region. Here, we combine these observations with new ultraviolet (UV) spectroscopy of \#016, some of the first data taken with the Cosmic Origins Spectrograph (COS) on the Hubble Space Telescope (HST), which reveal the star to have one of the highest wind terminal velocities seen to date in any massive star.

\section{OBSERVATIONS}

\subsection{UV Spectroscopy}

30 Dor $016\left(\alpha=05^{\mathrm{h}} 37^{\mathrm{m}} 08^{\mathrm{s}} .88, \delta=-69^{\circ} 07^{\prime} 20^{\prime} \cdot 36, \mathrm{~J} 2000\right)$ was observed with $H S T$-COS as part of the Servicing Mission Observatory Verification (SMOV) program in 2009 July using the G130M and G160M gratings in the far-UV channel. The data were obtained as part of a focus-check run early in the SMOV phase of COS operations so we do not have full wavelength coverage as the gratings were not moved. Outof-focus exposures $(<25 \%$ of the total $)$ were excluded when co-adding the spectra, with final exposure times of $5671 \mathrm{~s}$ $(\mathrm{G} 130 \mathrm{M})$ and $4511 \mathrm{~s}(\mathrm{G} 160 \mathrm{M})$. Estimates of the signal-to-noise ratio $(\mathrm{S} / \mathrm{N})$ in the combined spectrum are complicated by the large number of metallic absorption lines in the spectra of hot stars, particularly while the instrument is still undergoing full characterization. Here, we use selected continuum regions that are relatively free of lines to estimate the noise, yielding $\mathrm{S} / \mathrm{N} \gtrsim 40$. 


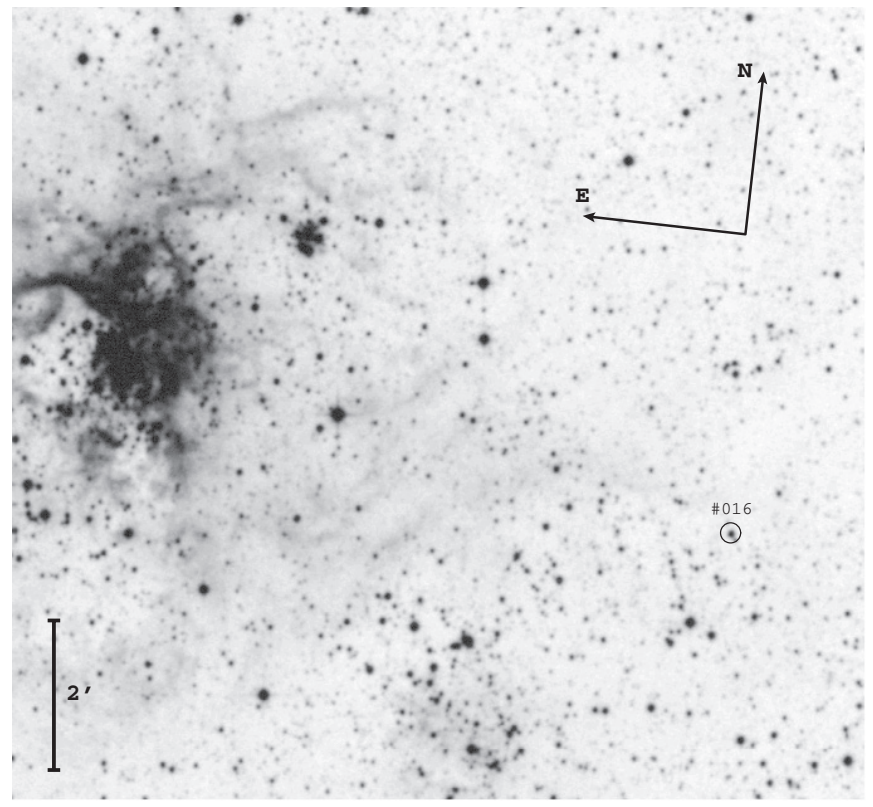

Figure 1. Digital Sky Survey "IR" ( $\sim$-band) image showing the location of 30 Dor 016 (encircled) relative to the 30 Dor complex.

Preliminary characterization of the COS on-orbit performance has found broad non-Gaussian wings in the line-spread function of the spectrograph owing to wavefront errors from the primary and secondary mirrors of the telescope (Ghavamian et al. 2009). This reduces the detection threshold for weak absorption features and modifies the profiles of saturated lines. To account for these effects, our models of the $\mathrm{C}$ IV line in Section 3 were convolved by the line-spread functions determined by the COS team.

The COS spectrum of \#016 is shown in Figure 2, together with an HST Space Telescope Imaging Spectrograph (STIS) E140M spectrum from program 9434 (P.I. Lauroesch) of HDE 269810 (Sk-67 211), classified as O2 III(f*) by Walborn et al. (2002). They are close twins, but with subtle differences-the most striking being the morphology of the CIV absorption and the P Cygni emission at He II $\lambda 1640$. Indeed, the C IV doublet in \#016 is saturated to a large velocity, indicative of a very fast stellar wind.

\subsection{Optical Data}

Optical spectroscopy of \#016 was obtained as part of two programs with $2 \mathrm{dF}$, before and after the AAOmega upgrade (Sharp et al. 2006). Three 2dF-AAOmega spectra were obtained on 2006 February 22-23 (P.I. JVL), with a 2dF spectrum obtained on 2004 December 8 (P.I. IDH), as summarized in Table 1.

The star was also observed as part of the Tarantula Survey (full details to be published elsewhere). Observations of \#016 with three of the standard FLAMES settings (LR02, LR03, and HR15N) were obtained over the period 2008 December 17-22. Pairs of exposures were taken in each observing block (OB), with three OBs observed at the LR02 and LR03 settings, and two at HR15N. Individual exposure times were $1815 \mathrm{~s}$ (LR02, LR03) and $2265 \mathrm{~s}(\mathrm{HR} 15 \mathrm{~N})$. The spectral coverage and resolution, as defined by the full width at half-maximum (FWHM) of the arc calibration lines, is given in Table 1.

The survey features repeat LR02 observations to detect radial velocity variables; for \#016 these were obtained on 2009 January
29, February 28, and October 8 (epochs 4, 5, and 6 in Table 1, respectively). The modified Julian Dates (MJD) for each epoch are listed in Table 1. Each co-added pair has an S/N > 100.

\subsubsection{Spectral Classification}

Informed by the apparent absence of detectable radialvelocity variations (Section 2.2.2), the individual LR02 and LR03 spectra were co-added, then merged in the overlap region, yielding an $\mathrm{S} / \mathrm{N}>350$.

The $\lambda \lambda 3950-4750 \AA$ region of \#016 is shown in Figure 2, compared with spectra of HDE 269810 and HD 93129A from Walborn et al. (2002). The most notable feature in the spectrum of \#016 is the strong N IV $\lambda 4058$ emission combined with an absence of $\mathrm{N}$ III $\lambda 4640$ emission, suggesting that the star is of the very earliest type in the existing classification framework. Also note the O IV $\lambda 4632$, Si IV $\lambda 4654$, and C IV $\lambda 4658$ emission, and the He II $\lambda 4686 \mathrm{P}$ Cygni profile. Accurate rectification of the $\lambda 4686$ and $\mathrm{H} \alpha$ regions is a notorious problem in observations of O-type stars, but we see broadened wings to the He II line in both manual and scripted rectifications.

As in the UV, the spectrum of \#016 is similar to that of HDE 269810. While the He II $\lambda 4686$ profile in \#016 suggests a somewhat more luminous star compared to HDE 269810, it is not as remarkable as the emission seen in HD 93129A (O2 If*; Walborn et al. 2002), leading to our intermediate classification of O2 III-If*.

\subsubsection{Stellar Radial Velocities}

There are relatively few optical lines for radial velocity analysis in such an early-type spectrum. The Balmer hydrogen lines and He II $\lambda 4686$ are strongly affected by the wind, so do not provide a true diagnostic of the stellar radial velocity. Overplotting of the data from each epoch (including those from $2 \mathrm{dF}$ ) revealed no significant velocity shifts nor broadening/ asymmetries in the lines, suggesting that a high-mass companion is not present and that \#016 is a prime candidate as an ejected, massive runaway from $\mathrm{R} 136$.

The XCORR routine in the DIPSO spectral analysis package (Howarth et al. 2004) was used to cross-correlate the co-added pairs of LR02 spectra with the first epoch of LR02 observations. The velocity offsets, $\Delta v_{\mathrm{r}}$ (see Table 1), are found from fits to the cross-correlation functions (CCFs) with Gaussian profiles generated using the DIPSO emission-line fitting (ELF) routine. Although the CCFs are not Gaussian, the ELF fits were restricted to the range $-100 \mathrm{~km} \mathrm{~s}^{-1}<\Delta v_{\mathrm{r}}<100 \mathrm{~km} \mathrm{~s}^{-1}$, yielding robust estimates with fitting errors of 3-4 $\mathrm{km} \mathrm{s}^{-1}$ (i.e., one tenth of a resolution element). From ELF fits to the He II $\lambda \lambda 4026,4200$, 4542 absorption, and Si Iv $\lambda \lambda 4098,4116$ emission lines, the mean heliocentric radial velocity for the first LR02 observations was $v_{\mathrm{r}}=191.8 \pm 1.4 \mathrm{~km} \mathrm{~s}^{-1}$.

Using the same methods we also cross-correlated the relevant calibration arcs to investigate the wavelength stability of FLAMES. The instrument is remarkably stable over all of our observations, with a largest offset of $\Delta v_{\mathrm{r}}=-0.3 \mathrm{~km} \mathrm{~s}^{-1}$, found between the arcs for epochs 1 and 6 (i.e., separated by 10 months).

Table 1 also lists cross-correlation results for the regions of the LR03 and 2dF-AAOmega spectra which overlap with the LR02 data; good agreement is found within the velocity resolution of the respective data. 

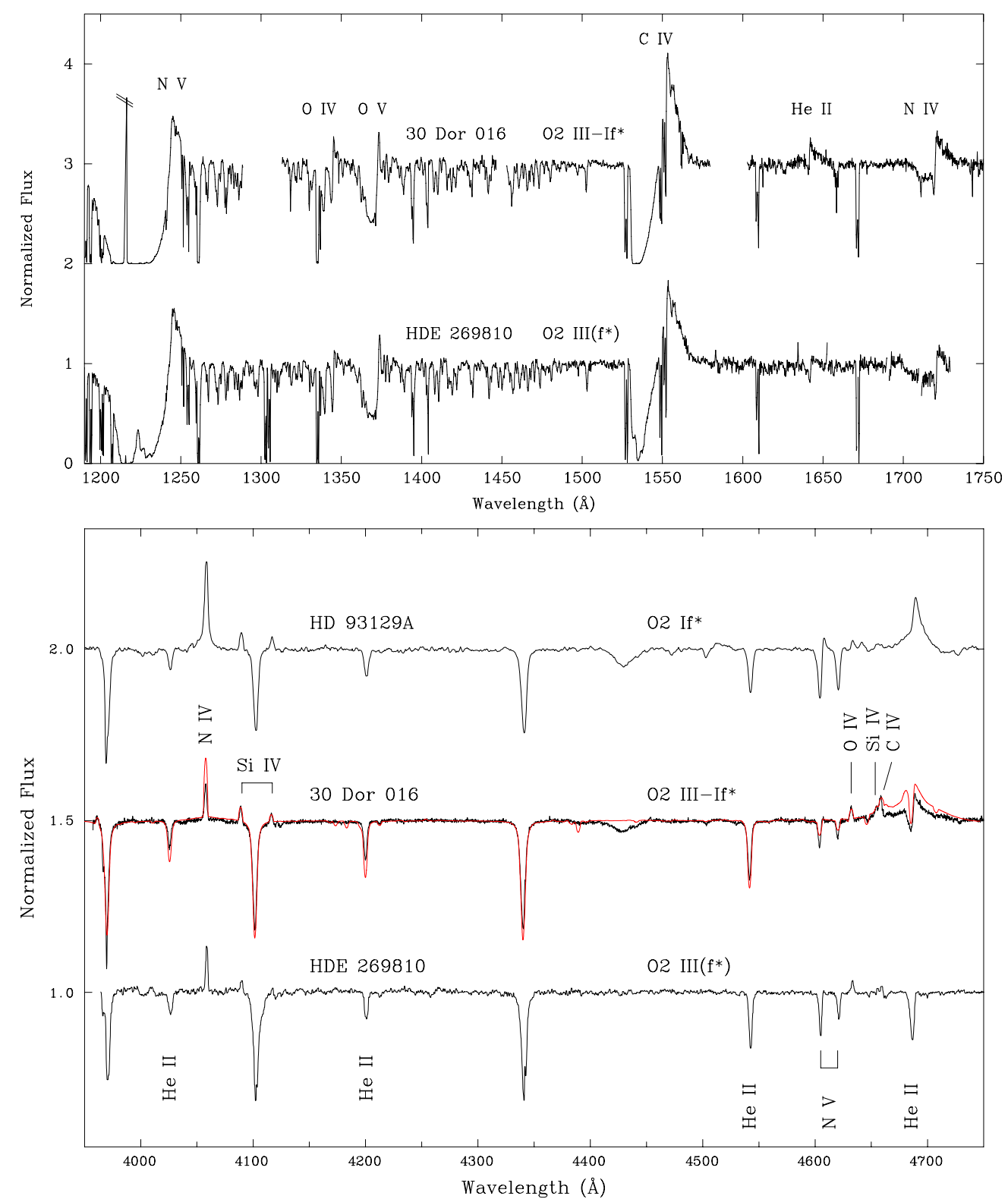

Figure 2. Upper panel: HST-COS spectrum of 30 Dor 016 and an HST-STIS spectrum of its near twin, HDE 269810 (both binned to $0.25 \AA$ ). The wind features identified are $\mathrm{N} v \lambda \lambda 1239-43$, O IV $\lambda \lambda 1339-44$, O v $\lambda 1371$, C IV $\lambda \lambda 1548-51$, He II $\lambda 1640$, and $\mathrm{N}_{\mathrm{IV}} \lambda 1718$. Lower panel: FLAMES spectrum of \#016, illustrating its place in the luminosity sequence at type O2 compared to HD 93129A and HDE 269810. Emission lines identified in \#016 are N IV $\lambda 4058$; Si IV $\lambda \lambda 4089$, 4116, 4654; O IV $\lambda 4632$; C IV $\lambda 4658$. Absorption lines identified in HDE 269810 are He II $\lambda \lambda 4026,4200,4542,4686 ; N$ v $\lambda \lambda 4604$, 4620. The CMFGEN model for \#016 is overplotted in red.

(A color version of this figure is available in the online journal.)

\subsubsection{Limits on a Binary Companion}

To investigate the confidence to which we can rule out a close massive companion to \#016, we used the method of Sana et al. (2009). In these calculations we adopted a $75 M_{\odot}$ primary, a bi-uniform period $(P)$ distribution (with $50 \%$ of the simulated systems with $0.3<\log P($ days $)<1$ and with $50 \%$ with $1<$ $\log P$ (days) $<3.5$ ), uniform distributions of eccentricities and mass ratios (between 0 and 0.9 , and 0.1 and 1.0, respectively), a random orientation in space, and a random periastron passage time.

Our observations allow us to rule out an O- or early B-type companion (with peak-to-peak variations $>20 \mathrm{~km} \mathrm{~s}^{-1}$ ) with a period in the range 2 days $<P<1 \mathrm{yr}$ at the $98.1 \%$ level. These results are only weakly dependent on the primary mass, with a variation of $\pm 1 \%$ for a primary in the range of $50-90 M_{\odot}$.

\section{TERMINAL WIND VELOCITY}

The Sobolev with exact integration (SEI) method was used to calculate UV line profiles to estimate $v_{\infty}$, the terminal wind velocity of \#016. We used the CCP7 SEI code ${ }^{10}$ which is based on the method from Lamers et al. (1987). The observed C IV $\lambda \lambda 1548-51$ doublet was blueshifted by $190 \mathrm{~km} \mathrm{~s}^{-1}$ (Section 2.2.2), then compared to the calculated profiles.

The code adopts a standard $\beta$-law to describe the acceleration of the stellar wind and includes a turbulence parameter, $v_{\mathrm{t}}$ (typically expressed as a fraction of $v_{\infty}$ ), to account for stochastic deviations from the velocity prescription. Line profiles were calculated over a range of values of $v_{\infty}, \beta$, and $v_{\mathrm{t}}$, then convolved by the line-spread function noted in Section 2.1. The best

\footnotetext{
10 http://ccp7.dur.ac.uk/library.html
} 
Table 1

Observational Epochs and Differential Radial Velocities

\begin{tabular}{|c|c|c|c|c|}
\hline $\begin{array}{l}\text { Instrument } \\
\text { and Setting }\end{array}$ & $\begin{array}{c}\text { FWHM } \\
(\AA)\end{array}$ & Epoch & MJD & $\begin{array}{c}\Delta v_{\mathrm{r}} \\
\left(\mathrm{km} \mathrm{s}^{-1}\right)\end{array}$ \\
\hline \multirow{6}{*}{$\begin{array}{l}\text { FLAMES-LR02 } \\
{[\lambda \lambda 3960-4564]}\end{array}$} & \multirow[t]{6}{*}{0.61} & 1 & $54,817.223$ & 0.0 \\
\hline & & 2 & $54,817.267$ & 1.7 \\
\hline & & 3 & $54,822.058$ & -1.8 \\
\hline & & 4 & $54,860.105$ & -1.7 \\
\hline & & 5 & $54,890.041$ & -1.7 \\
\hline & & 6 & $55,112.361$ & -2.0 \\
\hline \multirow{3}{*}{$\begin{array}{l}\text { FLAMES-LR03 } \\
{[\lambda \lambda 4499-5071]}\end{array}$} & \multirow[t]{3}{*}{0.56} & 1 & $54,818.243$ & 0.5 \\
\hline & & 2 & $54,818.287$ & -0.4 \\
\hline & & 3 & $54,818.330$ & -2.8 \\
\hline \multirow{2}{*}{$\begin{array}{l}\text { FLAMES-HR15N } \\
{[\lambda \lambda 6442-6817]}\end{array}$} & \multirow[t]{2}{*}{0.41} & 1 & $54,818.076$ & $\ldots$ \\
\hline & & 2 & $54,818.129$ & $\cdots$ \\
\hline 2dF-AAOmega & 1.0 & $\cdots$ & $53,788.442$ & 8.8 \\
\hline [1700B] & 1.0 & $\ldots$ & $53,788.490$ & -3.0 \\
\hline$[1500 \mathrm{~V}]$ & 1.25 & $\cdots$ & $53,789.430$ & -3.6 \\
\hline $2 \mathrm{dF}[1200 \mathrm{~B}]$ & 2.75 & $\ldots$ & $53,347.564$ & $\ldots$ \\
\hline
\end{tabular}

Notes. $\Delta v_{\mathrm{r}}$ results from cross-correlation with the first epoch LR02 observations.

$\chi$-squared fit (excluding the interstellar absorption features and the emission peak, where the SEI method is known to underestimate the flux by about $10 \%$ ) is obtained with $v_{\infty}=3450 \pm$ $50 \mathrm{~km} \mathrm{~s}^{-1}, \beta=0.75 \pm 0.1, v_{\mathrm{t}}=0.02 \pm 0.01 v_{\infty}$. The optical depth law parameters in the code $\left(\alpha_{1}\right.$ and $\left.\alpha_{2}\right)$ were set to 1 . For saturated lines these can be varied to obtain subtly different fits to the redward part of the absorption component but with no significant impact on the derived velocities. When $v_{\mathrm{t}}$ is relatively small, the value of $v_{\infty}$ obtained from fitting the line profile effectively corresponds to the blueward extent (i.e., $v_{\text {black }}$ ) of the saturated P Cygni profile.

This is one of the highest wind terminal velocities measured directly for an O-type star, behind only HDE $269810\left(v_{\infty}=\right.$ $3750 \mathrm{~km} \mathrm{~s}^{-1}$; Walborn et al. 1995) and R136a-608 ${ }^{11}$ (O3 If*, $v_{\infty}=3640 \mathrm{~km} \mathrm{~s}^{-1}$; Prinja \& Crowther 1998).

\section{QUANTITATIVE ANALYSIS}

Physical properties for a subset of O2-type stars were presented by Walborn et al. (2004). We employed similar methods to inform our discussion of \#016, using CMFGEN (Hillier $\&$ Miller 1998) which solves the radiative transfer equation in the co-moving frame, under the additional constraint of statistical equilibrium. The temperature structure follows from the assumption of radiative equilibrium. CMFGEN does not currently solve the momentum equation, so a density or velocity structure is required. The velocity for the supersonic part of the wind is parameterized with a classical $\beta$-type law $(\beta=0.75$, from Section 3 ), which is connected to a hydrostatic density structure at depth, such that the velocity and its gradient match at the interface. The subsonic density structure is set using a line-blanketed, plane-parallel TLUSTY model (v.200; Lanz \& Hubeny 2003) with $\log g=3.75$ (preferred over $\log g=4.0$ ). The atomic model is similar to that adopted by Walborn et al. (2004), including ions from $\mathrm{H}, \mathrm{He}, \mathrm{C}, \mathrm{N}, \mathrm{O}, \mathrm{Ne}, \mathrm{Si}, \mathrm{P}, \mathrm{S}, \mathrm{Ar}, \mathrm{Fe}$, and $\mathrm{Ni}$.

We assumed a depth-independent Doppler profile for all lines when solving for the atmospheric structure in the co-moving frame, and incoherent electron scattering and Stark broadening

11 Star 36 from Massey \& Hunter (1998). for hydrogen and helium lines was included. A uniform turbulence of $50 \mathrm{~km} \mathrm{~s}^{-1}$ was adopted in the calculation of the emergent spectrum in the observer's frame. Using the same methods as Walborn et al. (2010), a line-broadening parameter $(v \sin i)$ of $88 \pm 17 \mathrm{~km} \mathrm{~s}^{-1}$ was found from the optical spectra. Clumping was incorporated using a volume filling factor, $f$, as described by Hillier et al. (2003), with a typical value of $f=0.1$ resulting in a reduction in mass-loss rate by a factor of $\sqrt{(1 / f)} \sim 3$.

\subsection{Spectroscopic Results}

Based upon the observed UV to near-IR spectral energy distribution and the $\mathrm{H} \alpha$ and $\mathrm{He}$ II $\lambda \lambda 1640,4686$ wind features (with $v_{\infty}=3450 \mathrm{~km} \mathrm{~s}^{-1}$ ), we estimated the stellar temperature, luminosity and mass-loss rate of \#016 simultaneously.

For an adopted LMC distance of $49 \mathrm{kpc}$ (distance modulus $=$ 18.45), the UV spectrophotometry and optical ${ }^{12}$ and near-IR photometry ${ }^{13}$ of \#016 can be reproduced with interstellar extinctions of $E(B-V)=0.07$ (foreground) and 0.27 (LMC), using standard Galactic and LMC (field) extinction laws (Seaton 1979; Howarth 1983) and a stellar luminosity of $L_{*}=1.2 \times 10^{6} L_{\odot}$, as shown in Figure 3. The relatively large local extinction is supported by the strong $\lambda 4428$ diffuse interstellar band, which is at a comparable radial velocity to \#016.

In the absence of measurable He I $\lambda 4471$ absorption, a combination of optical nitrogen lines ( $\mathrm{N}$ IV $\lambda 4058, \mathrm{~N} v \lambda \lambda 4603$ 20) and UV oxygen lines ( $\mathrm{IV} \lambda \lambda 1339-44, \mathrm{O} v \lambda 1371$ ) was used to derive an estimated effective temperature $\left(T_{\text {eff }}\right)$ of $50 \mathrm{kK}$ for \#016. This is a compromise between the available diagnostics - the optical $\mathrm{N} \mathrm{v}$ lines favor a temperature some $5 \%-10 \%$ higher, while the UV O IV suggests $10 \%$ lower-but the strong N IV $\lambda 1718$ P Cygni profile and negligible $S$ v $\lambda 1501$ line both firmly support $T_{\text {eff }}=50 \mathrm{kK}$. Given the adopted wind parameters, a slightly greater broadening profile was preferred ( $v \sin i \sim 150 \mathrm{~km} \mathrm{~s}^{-1}$ ) in the final model than determined above.

A range of nitrogen abundances were considered, adopting $X_{\mathrm{N}}=0.08 \%$ by mass, which is substantially greater than the interstellar abundance in the LMC $(0.016 \%$; Russell \& Dopita $1990)$. For carbon and oxygen we adopt $X_{\mathrm{C}}=0.08 \%$ and $X_{\mathrm{O}}=0.2 \%$ by mass, slightly below the results from Dufour (1984) and Russell \& Dopita (1990) for the interstellar medium ( $0.095 \%$ and $0.35 \%$, respectively), although the UV oxygen diagnostics are relatively insensitive to abundance changes.

The adopted model is shown in Figures 2 and 3, including allowance for an LMC neutral hydrogen column density of $10^{21.6} \mathrm{~cm}^{-2}$. In general, the fits are satisfactory, with a few exceptions. The UV P Cygni absorption troughs of O v $\lambda 1371$ and N IV $\lambda 1718$ extend too far blueward, although the majority of the "iron forest" (Fe V-VI) features are well matched. As with the temperature, the adopted mass-loss rate $\left(\dot{M}=3 \times 10^{-6} M_{\odot} \mathrm{yr}^{-1}\right)$ is a compromise between the available diagnostics (He II $\lambda \lambda 1640,4686$ and $\mathrm{H} \alpha$ ). Weak metallic lines in the optical are, in general, reproduced satisfactorily, e.g., Si IV $\lambda \lambda 4089-4116$, O IV $\lambda 4632$, and the blend of C IV $\lambda 4658$ and $\mathrm{O}$ IV $\lambda 4654,4663$. A more detailed treatment of \#016 (including the role of clumping, line-broadening, and the wind acceleration and turbulence) is beyond the scope of this Letter and will be presented elsewhere.

A summary of physical and wind parameters of \#016 is provided in Table 2, compared with those for HDE 269810

\footnotetext{
$12 U=12.62, B=13.53, V=13.49, R=13.43$ (Massey 2002).

$13 J=13.38, H=13.35, K_{\mathrm{s}}=13.36$ (Two Micron All Sky Survey; Skrutskie et al. 2006).
} 


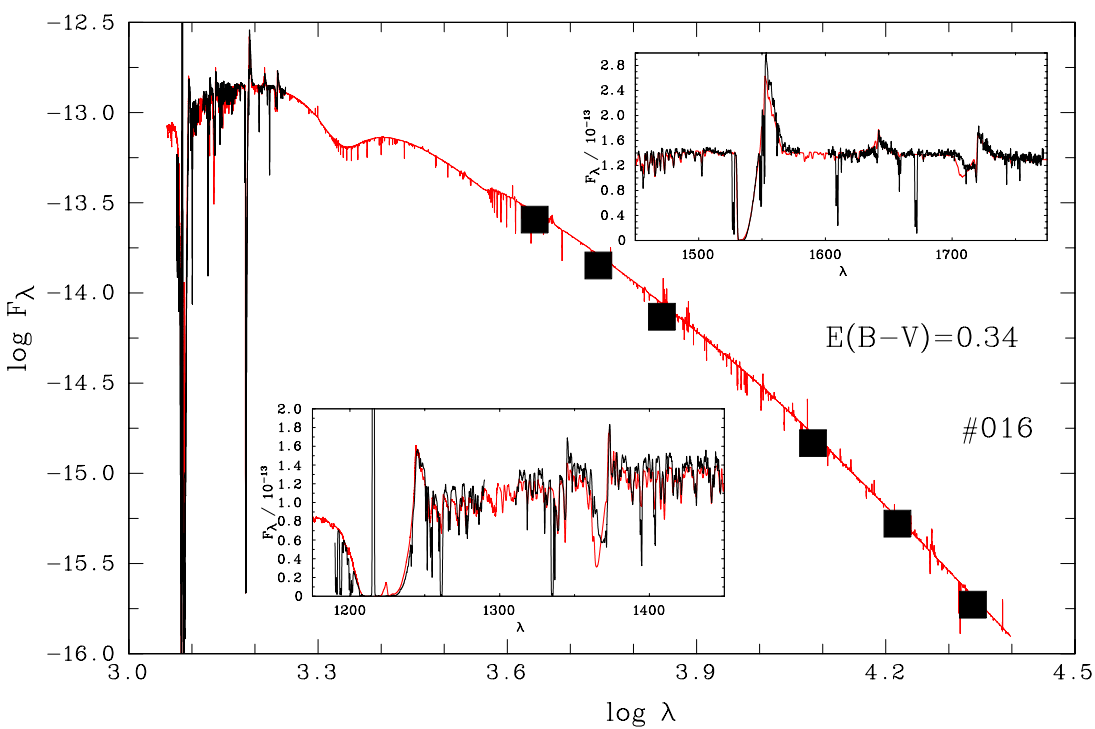

Figure 3. CMFGEN fits (red) to the flux-calibrated UV and photometric observations (black lines/squares), used to determine the interstellar extinction toward 30 Dor 016. Insets are expanded plots of the fits to the HST-COS spectra.

(A color version of this figure is available in the online journal.)

Table 2

Physical and Wind Properties of 30 Dor 016 and HDE 269810

\begin{tabular}{lcc}
\hline \multicolumn{1}{c}{ Star } & 30 Dor 016 & HDE 269810 \\
\hline Sp type & O2 III-If* & O2 III(f*) \\
$T_{\text {eff }}(\mathrm{kK})$ & 50 & 52.5 \\
$\log L / L_{\odot}$ & 6.08 & 6.34 \\
$M_{\odot}$ & $90:$ & $130:$ \\
$\log g(\mathrm{cgs})$ & 3.75 & 4.0 \\
$v_{\infty}\left(\mathrm{km} \mathrm{s}^{-1}\right)$ & 3450 & 3750 \\
$\dot{M}\left(M_{\odot} \mathrm{yr}^{-1}\right)$ & $10^{-5.5}$ & $10^{-5.7}$ \\
$M_{\mathrm{V}}\left(\mathrm{mag}^{-1}\right)$ & -6.0 & -6.6 \\
$\mathrm{He} / \mathrm{H}$ & $\leqslant 0.1$ & $\leqslant 0.1$ \\
$X_{\mathrm{C}}(\%)$ & 0.08 & 0.06 \\
$X_{\mathrm{N}}(\%)$ & 0.08 & 0.03 \\
$X_{\mathrm{O}}(\%)$ & 0.2 & 0.3 \\
\hline
\end{tabular}

Notes. Mass estimates are obtained from non-rotating evolutionary models at LMC metallicity (R. Hirschi 2010, private communication). Metal abundances are reliable to within 50\% $(\mathrm{N})$, or a factor of $2(\mathrm{C}$ and $\mathrm{O})$.

from Walborn et al. (2004). We have also compared our results with LMC-metallicity, non-rotating evolutionary predictions (R. Hirschi 2010, private communication) calculated with the mass-loss recipe of Vink et al. (2001). This approach is supported by our derived mass-loss rate, which agrees with theoretical predictions to within $\sim 0.05$ dex for \#016. We obtain the best agreement for a stellar mass of $\sim 90 M_{\odot}$ at an age of $\sim 1 \mathrm{Myr}$, corresponding to $\log g \sim 4$.0. In contrast, the spectroscopic gravity suggests a considerably lower mass of $\sim 50 M_{\odot}$. Using the evolutionary mass to calculate the escape velocity, $v_{\text {esc }}$, our measured terminal velocity is in excellent agreement with the expected value using the empirical relation $v_{\infty} \sim 2.6 v_{\text {esc }}$ (Lamers et al. 1995), as well as new predictions for the velocity structure of massive star winds (L. Muijres et al. 2010, in preparation).

\section{30 DOR 016 AS A MASSIVE RUNAWAY}

30 Dor 016 is located at the end of an extended rarefied filament of nebular gas, at a radial distance of $\sim 8.25$ (120 pc in projection) west-southwest of R136, and 4.75 (70 pc) from the less dense cluster NGC 2060. The [O III] emission superimposed on the stellar spectrum has a peak velocity of $\sim 275 \mathrm{~km} \mathrm{~s}^{-1}$, with some asymmetry toward slightly lower velocities but with no evidence for a second component matching the velocity of \#016. This suggests \#016 did not form locally and is a bona fide runaway star.

Further analysis of the stellar and gas dynamics in this region is warranted to complete our picture of the origins of \#016, e.g., van Loon \& Zijlstra (2001) found molecular gas to the southeast of R136 at a comparable radial velocity to the star. At present, we lack proper motions at useful precision to constrain its transverse velocity: $\mu_{\alpha}=1.2 \pm 5.9, \mu_{\delta}=1.5 \pm 5.9 \mathrm{mas} \mathrm{yr}^{-1}$ from UCAC2; $\mu_{\alpha}=2.8 \pm 3.8, \mu_{\delta}=-4.3 \pm 3.8 \mathrm{mas} \mathrm{yr}^{-1}$ from UCAC3 (and flagged as uncertain; Zacharias et al. 2010). HST imaging of this region would provide much improved constraints (e.g., the precision achieved by Kallivayalil et al. 2006), and in the longer term, the Gaia mission should deliver transverse velocities in the LMC to a few $\mathrm{km} \mathrm{s}^{-1}$.

There are two other O2-type stars (Sk-68 137 and BI 253) to the north and northwest of 30 Dor, which were proposed as runaways by Walborn et al. (2002). BI 253 has also been observed within the Tarantula Survey, but from preliminary inspection of its spectra, its radial velocity appears consistent with this part of the LMC. The case of \#016 is also reminiscent of the discovery of N11-026 (O2.5 III ( $\left.\left.\mathrm{f}^{*}\right)\right)$, suggested as a runaway by Evans et al. (2006).

Runaway stars are thought to result from either dynamical interaction in massive dense clusters, or via a kick from a supernova explosion in a binary system, with the more massive star exploding first (see, e.g., Gvaramadze et al. 2009, and references therein). It is generally accepted that R136 is sufficiently young (1-2 Myr) that its most massive stars have yet to explode as supernovae. This implies that, if from R136, \#016 must have been ejected through dynamical interaction, one of the clearest cases to date in support of this mechanism. This is vitally important as dynamical interactions in massive clusters are thought to be a possible mechanism for producing stellar mergers and very massive stars (with masses $>140 M_{\odot}$ ) which might subsequently end their lives as pair-instability supernovae, 
of broader relevance to the early ages of the universe when such massive stars are thought to be common (e.g., Heger et al. 2003; Gal-Yam et al. 2009).

We thank Alex de Koter, Paco Najarro, and the referee, Hans Zinnecker, for their helpful comments.

\section{REFERENCES}

Bosch, G., Terlevich, E., \& Terlevich, R. 2009, AJ, 137, 3437

Dufour, R. J. 1984, in IAU Symp. 108, Structure and Evolution of the Magellanic Clouds, ed. S. van den Bergh \& K. S. de Boer (Dordrecht: Reidel), 353

Evans, C. J., Lennon, D. J., Smartt, S. J., \& Trundle, C. 2006, A\&A, 456, 623

Evans, C. J., et al. 2010, in IAU Symp. 266, Star Clusters: Basic Galactic Building Blocks throughout Time and Space, ed. R. de Grijs \& J. R. D. Lépine (Cambridge: Cambridge Univ. Press), 35

Gal-Yam, A., et al. 2009, Nature, 462, 624

Ghavamian, P., et al. 2009, COS Instrument Science Report 2009-01(v1) (Baltimore, MD: STSci), http://www.stsci.edu/hst/cos/documents/isrs/ ISR2009_01(v1).pdf

Gvaramadze, V. V., Gualandris, A., \& Portegies Zwart, S. 2009, MNRAS, 396, 570

Heger, A., Fryer, C. L., Woosley, S. E., Langer, N., \& Hartmann, D. H. 2003, ApJ, 591,288

Hillier, D. J., \& Miller, D. L. 1998, ApJ, 496, 407

Hillier, D. J., et al. 2003, ApJ, 588, 1039
Howarth, I. D. 1983, MNRAS, 203, 301

Howarth, I. D., Murray, J., Mills, D., \& Berry, D. S. 2004, Starlink User Note 50.24 (Swindon: PPARC), http://www.starlink.rl.ac.uk/star/docs/sun50.htx/ sun50.html

Kallivayalil, N., van der Marel, R. P., \& Alcock, C. 2006, ApJ, 638, 772

Lamers, H. J. G. L. M., Cerruti-Sola, M., \& Perinotto, M. 1987, ApJ, 314, 726

Lamers, H. J. G. L. M., Snow, T. P., \& Lindholm, D. M. 1995, ApJ, 455, 269

Lanz, T., \& Hubeny, I. 2003, ApJS, 146, 417

Massey, P. 2002, ApJS, 141, 81

Massey, P., \& Hunter, D. A. 1998, ApJ, 493, 180

Melnick, J. 1985, A\&A, 153, 235

Prinja, R. K., \& Crowther, P. A. 1998, MNRAS, 300, 828

Russell, S. C., \& Dopita, M. A. 1990, ApJS, 74, 93

Sana, H., Gosset, E., \& Evans, C. J. 2009, MNRAS, 400, 1479

Seaton, M. J. 1979, MNRAS, 187, 73

Sharp, R., et al. 2006, Proc. SPIE, 6269, 14

Skrutskie, M. F., et al. 2006, AJ, 131, 1163

Smartt, S. J. 2009, ARA\&A, 47, 63

van Loon, J. Th., \& Zijlstra, A. A. 2001, ApJ, 547, L61

Vink, J. S., de Koter, A., \& Lamers, H. J. G. L. M. 2001, A\&A, 369, 574

Walborn, N. R. 1971, ApJ, 167, L31

Walborn, N. R., \& Blades, J. C. 1997, ApJS, 112, 457

Walborn, N. R., et al. 1995, PASP, 107, 104

Walborn, N. R., et al. 2002, AJ, 123, 2754

Walborn, N. R., et al. 2004, ApJ, 608, 1028

Walborn, N. R., et al. 2010, AJ, 139, 1283

Zacharias, N., et al. 2010, AJ, in press (arXiv:1003.2136) 